\title{
A CHEWABLE BREATH-BOOST FOR PATIENTS
}

CB12 boost is a brand new, patented chewing gum from the developers of CB12 mouth rinse, the safe breath solution that lasts for 12 hours.

CB12 boost is a sugar-free, twolayer chewing gum with triple action; it

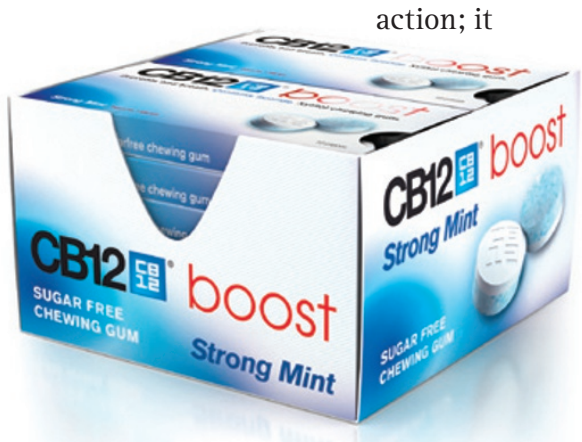

contains zinc to prevent bad breath, xylitol to reduce plaque and sodium fluoride to strengthen teeth and prevent caries. It prevents halitosis and provides instant breath freshness when it is most needed. The unique combination of active ingredients protects users while offering instant refreshment with a fresh, minty flavour. As chewing gum is the preferred breath freshener over other alternatives, CB12 boost meets the demand, with research revealing that $75 \%$ of people feel the need to freshen their breath several times a week.

Recommend CB12 boost, used in combination with CB12 mouth rinse, to fulfil all of your patients' needs at any time of day.

For more information about CB12 and how it can benefit your patients, visit www.cb12.co.uk.

\section{A BARRIER AGAINST CONTAMINATED FLUIDS}

As one of their many ways of saying Happy New Year, The Dental Directory is releasing a special $2+1$ Flyer for UnoDent protective eyewear.

The UnoDent range of protective glasses helps ensure the safety of staff members and patients throughout all dental procedures.

UnoDent glasses guarantee a protective barrier against contaminated fluids and other residue and offer a range of lens types from anti-fog to scratch resistant. Their sleek designs also provide relaxed fits for stylish and comfortable protection.
To keep your UnoDent glasses smudge-free, a new glasses cleaner station is also now available from The Dental Directory.

The $2+1$ offer is only valid from January to February 2014, so place your orders for UnoDent Glasses from The Dental Directory today!

For more information about UnoDent glasses and the special offer call 0800585586 or visit www.dental-directory.co.uk.

\section{DUAL-CURING UNIVERSAL ADHESIVE}

Futurabond $U$ is the only true dual-curing universal adhesive offering usage in a disposable applicator. Besides the incredibly simple handling of SingleDose the new Futurabond U offers practitioners an outstanding range of options for application, as much with regard to indications as to selection of the etching technique (self-etch, selective-etch or total-etch) or the curing mode. This universal adhesive is fully compatible with all light-curing, dual-curing and self-curing methacrylate-based composites and is suitable for both direct and indirect restorations. Applied in a single layer, Futurabond U creates a strong bond to enamel and dentine, thus ensuring a durable, gap-free bond between the dental hard tissue and the restorative material. At the same time it offers firm adhesion to different materials such as metal, zirconium/aluminium oxide and silicate ceramics without any additional primer.

Special offer: buy one pack of 200 SingleDose and get a Trial Pack of 20 SingleDose for free, all with a payback guarantee. This offer is available until 31 March 2014. Ask your VOCO dental consultant or contact your dental dealer. Emailinfo-uk@voco.com. Visit www.voco.com.

\section{REMOVE DIRT THAT OTHER} CLOTHS LEAVE BEHIND

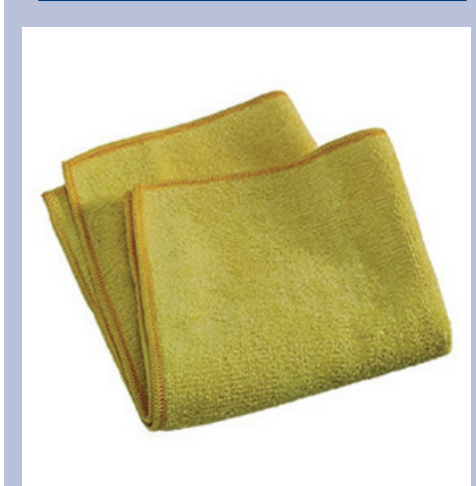

Unlike conventional cloths, Evident's range of re-usable, washable microfibre cloths, e-cloth Professional have 30,000 fibres per square inch and more than 500 million fibre strands per cloth. These special fibres are capable of entangling and thus remove a wide range of pathogenic particles. When damp, its capillary action rapidly draws contamination into the fibre gaps, making it effective in removing grease, dirt and bacteria from surfaces, which other cloths leave behind. In addition, every individual cloth is impregnated with antibacterial nanosilver, which remains active throughout the life of the cloth.

Successfully removing over 99\% of bacteria from surfaces without the need for harsh, needlessly expensive cleaning products, e-cloth Professional is extremely cost-effective and eco-friendly, helping save time and money just by using water. Plus these cloths are washable (guaranteed up to 1,000 washes) so can be reused again and again. Following National Patient Safety Agency guidelines (supplied on request) e-cloth Professional cloths are available in packs of ten in yellow, blue, red or green.

For more information contact Evident free on 0500321111 or visit www.evident.co.uk. 